\title{
Prognostic Values of Alpha-Fetoprotein and Des-Gamma-Carboxyprothrombin in Hepatocellular Carcinoma in China: An Analysis of 4792 Patients
}

\author{
Yang-Xun Pan (D) ${ }^{1-3, *}$ \\ Xu-Qi Sun ${ }^{1,2, *}$ \\ $\mathrm{Zi}-\mathrm{Li} \mathrm{Hu}{ }^{1,2, *}$ \\ Wa Xie ${ }^{1,2}$ \\ $\mathrm{Ke}-\mathrm{Xin} \mathrm{Nie}^{4}$ \\ Ai-Ping Fang ${ }^{5}$ \\ Ying-Yao Zhang ${ }^{4}$ \\ Yi-Zhen $\mathrm{Fu}^{\mathrm{I}, 2}$ \\ Jin-Bin Chen (D) ${ }^{1,2}$ \\ Jun-Cheng Wang ${ }^{1,2}$ \\ Xin Wang ${ }^{1,2}$ \\ Yao-Jun Zhang (D) ${ }^{1,2}$ \\ Dan-Dan Hu ${ }^{1,2}$ \\ Min-Shan Chen ${ }^{1,2}$ \\ 'Collaborative Innovation Center for \\ Cancer Medicine, State Key Laboratory of \\ Oncology in South China, Sun Yat-Sen \\ University Cancer Center, Guangzhou, \\ Guangdong, 510060, People's Republic of \\ China; ${ }^{2}$ Department of Liver Surgery, Sun \\ Yat-Sen University Cancer Center, \\ Guangzhou, Guangdong, 510060, People's \\ Republic of China; ${ }^{3}$ Department of \\ Oncology-Pathology, Karolinska Institutet, \\ Solna, 17165, Sweden; ${ }^{4}$ Real World Study \\ Team, BaseBit.ai, Shanghai, 200050, People's \\ Republic of China; ${ }^{5}$ Department of \\ Nutrition, School of Public Health, Sun Yat- \\ sen University, Guangzhou, 510080, People's \\ Republic of China \\ *These authors contributed equally to this \\ work
}

Correspondence: Dan-Dan Hu; Min-Shan Chen

Department of Liver Surgery, Sun Yat-Sen University Cancer Center, 65I Dongfeng

Road East, Guangzhou, Guangdong, 510060 , People's Republic of China

Tel +86-20-87343 II 5; +86-20-87343 III

Fax +86-20-87343585; +86-20-8734358

Email hudd@sysucc.org.cn;

chenmsh@sysucc.org.cn
Background: The importance of alpha-fetoprotein (AFP) and des-gammacarboxyprothrombin (DCP) in hepatocellular carcinoma (HCC) has been studied extensively in Japan, where hepatitis $\mathrm{C}$ virus is the predominant aetiology of HCC. The clinical profiles of HCC regarding the state of AFP and DCP in a hepatitis B virus epidemic area have not been comprehensively investigated, and the value of these tumour markers in evaluating the response to treatment and the detection of recurrence has yet to be determined.

Patients and Methods: A total of 4792 patients treated in our centre were continuously analysed regarding accessible AFP and DCP data pre- and posttreatment. Baseline characteristics were summarized, and comparisons of progression-free survival (PFS) and overall survival (OS) rates were made independently. The prognostic significance of each factor was tested with the Cox proportional hazards model. Patients who had AFP and DCP data pretreatment, pre- and posttreatment, and those who were continuously monitored more than twice were analysed separately.

Results: A total of 2600 patients (53.4\%) were positive for AFP and DCP; 362 (7.6\%) and 1211 (25.3\%) patients were AFP- or DCP-positive, respectively, and 619 patients $(12.9 \%)$ were negative for both AFP and DCP. Patients in the AFP single-positive or double-negative groups had the best OS $(P<0.001)$. Patients with less than 50\% responses in AFP and DCP after treatments suffered from worse prognostic survival $(P<0.001)$. In the multivariate analysis, elevated AFP and DCP were identified as independent prognostic factors of PFS and OS. In addition, different tumour markers were related to different clinical and pathological traits.

Conclusion: The present study comprehensively explored the clinical value of classical tumour markers for HCC using the "point-to-line" method. Positivity of pretreatment AFP and DCP or less than $50 \%$ treatment response rates exhibited more aggressive HCC, resulting in poor PFS and OS in HCC patients.

Keywords: alpha-fetoprotein, des-gamma-carboxy prothrombin, hepatocellular carcinoma, clinical features

\section{Introduction}

Hepatocellular carcinoma (HCC) is the sixth most common cancer worldwide and the fourth leading cause of cancer-related death for years. ${ }^{1}$ For diagnosis and treatment, serological tests are important for early diagnosis, monitoring response to treatment, and early detection of recurrence. Alpha-fetoprotein (AFP) combined with imaging studies is considered a reliable combination for early diagnosis of HCC according to guidelines of the National Comprehensive Cancer Network 
(NCCN) and the Asian Pacific Association for the Study of the Liver (APASL). ${ }^{2,3}$ The sensitivity and specificity of AFP vary from $4.0 \%$ to $31.0 \%$ and $76.0 \%$ to $100.0 \%$, respectively, with a cut-off value of $200 \mathrm{ng} / \mathrm{mL}$ for $\mathrm{HCC}$ smaller than $5 \mathrm{~cm}$ in diameter. ${ }^{4}$ Moreover, AFP also plays critical roles in population-based screening and surveillance.

Although AFP has been widely accepted as a first-line tumour marker for HCC for many years, other tumour markers have shown potential diagnostic efficacy in HCC. Des-gamma-carboxyprothrombin (DCP), also known as prothrombin induced by vitamin $\mathrm{K}$ absence-II (PIVKA-II), is an abnormal prothrombin protein that is significantly increased in the serum of HCC patients. Based on previous studies, DCP was recognized as not only a highly specific marker but also a predictor of prognosis for HCC patients. ${ }^{6}$ DCP enriches the panel of serologic tests and provides more possibilities for acquiring serologic evidence for HCC diagnosis and clinical decisions.

Recent studies have demonstrated better diagnostic and prognostic value by combining AFP and DCP in HCC management. Chon et al divided HCC patients into 2 groups (double positive or not) according to their profiles of preoperative AFP and DCP. ${ }^{7}$ They reported that the double-positive group had a higher recurrence rate and shorter disease-free survival even though they received more curative treatment than the other group. Moreover, Kamiyama et al employed the multiplication of AFP and DCP (AP-factor) to evaluate the recurrence of $\mathrm{HCC}$ patients after hepatectomy. ${ }^{8}$ The AP factor showed a close relationship to tumour differentiation and microscopic vascular invasion, which in turn affected survival and disease recurrence. Following these studies, researchers in Japan performed substantial studies aiming to describe the relationship between tumour markers and the tumour biological behaviour of HCC patients. However, given that the main aetiology of HCC in Japan is hepatitis $\mathrm{C}$ virus (HCV) infection, the conditions of AFP and DCP in the hepatitis B virus (HBV) epidemic area have yet to be studied. To our knowledge, few studies have inspected the prognostic values and survival outcomes of pretreated levels of AFP and DCP in HCC patients from China in a large patient population. Moreover, the dynamic changes in AFP and DCP after therapies are seldom described due to the lack of follow-up data.

In this study, we retrospectively investigated the AFP and DCP levels of 4792 HCC patients in a Chinese cancer centre and analysed the oncological outcomes regarding different tumour marker profiles. Furthermore, a series of subset analyses was performed based on the available AFP and DCP data after treatments, to further explore the value of monitoring AFP and DCP continuously.

\section{Patients and Methods}

\section{Patients}

Patients who were initially diagnosed with HCC predominantly based on pathological examinations were retrospectively analysed from November 2016 to December 2019 at Sun Yat-sen University Cancer Center (SYSUCC). The workflow of enrolment and analysis for this study is illustrated in detail in Figure 1. A total of 4792 of 17,397 patients who had undergone measurement of the levels of two pretreated tumour markers, AFP and DCP, were included in the initial analysis, and then patients with positive pretreatment AFP and DCP who also had posttreatment AFP and DCP were employed for the AFP and DCP response analysis. Finally, patients who were continuously monitored for AFP and DCP more than twice were included in the long-term follow-up analysis. Exclusion criteria included other types of liver cancer.

For the initial analysis, patients were divided into 4 groups according to AFP and DCP levels within one month before treatment. Patients who were both AFPand DCP-positive (AFP $\geq 25 \mathrm{ng} / \mathrm{mL}, \mathrm{DCP} \geq 40 \mathrm{mAU} / \mathrm{mL}$ ) were assigned to group $\mathrm{A}$, those who were either AFP positive or DCP positive were assigned to group B or C, respectively, and the remainder were assigned to group D. For the AFP and DCP response analysis, patients were also divided into 4 groups based on the AFP and DCP response the first month after treatment. ${ }^{9,10}$ Patients who showed over $50 \%$ decline for both AFP and DCP after treatments $(\triangle \mathrm{AFP}>50 \% ; \Delta \mathrm{DCP}>50 \%)$ were assigned as group A, either AFP or DCP decreased more than $50 \%$ compared to pretreatment were grouped as B $(\triangle \mathrm{AFP}>50 \% ; \Delta \mathrm{DCP} \leq 50 \%)$ or $\mathrm{C}(\triangle \mathrm{AFP} \leq 50 \%$; $\triangle \mathrm{DCP}>50 \%)$, respectively, and the remainder were set as group $\mathrm{D}(\triangle \mathrm{AFP} \leq 50 \% ; \triangle \mathrm{DCP} \leq 50 \%)$. The final long-term follow-up analysis included patients who had AFP and DCP monitored continuously after treatment until the last follow-up or reported progression. Other clinical data, including demographic characteristics, laboratory tests, imaging studies, pathological traits, tumour staging, and survival statistics, were also collected. 
This retrospective study was approved by the Institutional Review Board of SYSUCC (B2020-350-01), and informed consent was waived. However, consent for relevant procedures and the use of data for research purposes were obtained from patients before treatment. This study was conducted in accordance with the Declaration of Helsinki.

\section{Tumour Marker Measurement}

The serum AFP and DCP levels were measured by electrochemiluminescence immunoassay using the Roche Cobas E602 system (Roche Diagnostics GmbH, Mannheim, Germany) and the ARCHITECT i2000 immunoassay analyser (Abbott Laboratories, North Chicago, IL) per the manufacturer's instructions, respectively. The cut-off value of AFP for $\mathrm{HCC}$ was set at $25 \mathrm{ng} / \mathrm{mL}$ according to a previous study. ${ }^{11}$ The cut-off value of DCP was determined to be 40 $\mathrm{mAU} / \mathrm{mL}$ for the differentiation of HCC and nonmalignant liver disease based on previous research. ${ }^{12}$

\section{Follow-Up After Treatment}

Patients were scheduled to receive dynamic computed tomography (CT) scans or magnetic resonance imaging (MRI), chest radiography and laboratory tests 1 month after treatment, every 3 months during the first 2 years, and every 4-6 months thereafter. If possible progression was suspected based on radiological features, such as an abnormality on residual liver or chest or clinical symptoms (eg, severe headache or neurological symptoms, bone pain), further tests and treatments would also be performed.

\section{Definitions of Outcomes}

Progression-free survival (PFS) was defined as the interval between initial treatment and the date of local tumour progression, intrahepatic recurrence, or extrahepatic recurrence. Overall survival (OS) was defined as the interval between the first treatment and either the date of death or the latest follow-up visit, with a final evaluation on May 31, 2020.

\section{Statistical Analysis}

Independent-sample $t$-tests, chi-square tests or Fisher's exact tests were used to analyse the differences in clinical, radiological, and pathological characteristics between groups of interest. Consecutive data are presented as the mean \pm standard deviation (SD). The survival curves were established by the Kaplan-Meier method with the Log rank test. Univariate analysis was performed including the following factors: age, sex, presence of HBV surface antigen, albumin (ALB), presence of ascites, total bilirubin (TBIL), prothrombin times (PT), activated partial thromboplastin time (APTT), tumour number, tumour size, presence of vascular invasion, presence of extrahepatic metastasis, pretreatment AFP $(<25 \mathrm{ng} / \mathrm{mL}$ or $\geq 25 \mathrm{ng} / \mathrm{mL})$ and pretreatment DCP $(<40$ $\mathrm{mAU} / \mathrm{mL}$ or $\geq 40 \mathrm{mAU} / \mathrm{mL}$ ). Possible risk factors with a $P$ value of 0.10 or less on univariate analysis and complete clinical data were subjected to multivariate analyses using the Cox proportional hazards regression method with stepwise variable selection to identify independent factors associated with survival and disease progression rates. Statistical analysis was performed using R version 3.5.1 (R Foundation for Statistical Computing, Vienna, Austria). Statistical tests were 2-sided, and $P<0.050$ was considered significant.

\section{Results}

\section{Patient Demographics and Tumour Characteristics}

In total, 4792, 1956, and 985 patients were enrolled in the three series of analyses, respectively (Figure 1). The baseline demographics and tumour characteristics of 4792 patients are presented in Table S1, and the baseline AFP and DCP profiles are shown in Table 1. HBV infection was the most common aetiology of $\mathrm{HCC}(3563,74.4 \%)$, the majority of patients were male $(4153,86.7 \%)$, and half of them received radical therapies $(2379,49.6 \%)$. For laboratory tests, most patients were at Child-Pugh class A (2209, 46.1\%) and at an acceptable condition before treatment regarding liver profiles. The distributions of AFP and DCP for 4792 patients are presented as a dot plot in Figure S1A. The median AFP and DCP levels were $58.2 \mathrm{ng} / \mathrm{mL}$ and $279 \mathrm{mAU} / \mathrm{mL}$, respectively. In detail, 2962 (61.8\%) and 3811 (79.5\%) patients showed positivity for AFP and DCP before treatment, respectively. Among 1830/4792 (38.2\%) patients with negative AFP results, 1211/1830 (66.2\%) had positive DCP, which provided serological evidence for the diagnosis of HCC.

There were 2600 (54.3\%), 362 (7.6\%), 1211 (25.3\%) and $619(12.9 \%)$ patients in groups A, B, C and D, respectively (Table 1). Patients in group A who were AFP and DCP double positive had the highest proportions of more than two tumours $(1001,38.5 \%)$, larger than $5 \mathrm{~cm}$ tumour size $(884,33.4 \%)$, vascular invasion $(330,12.7 \%)$ and 


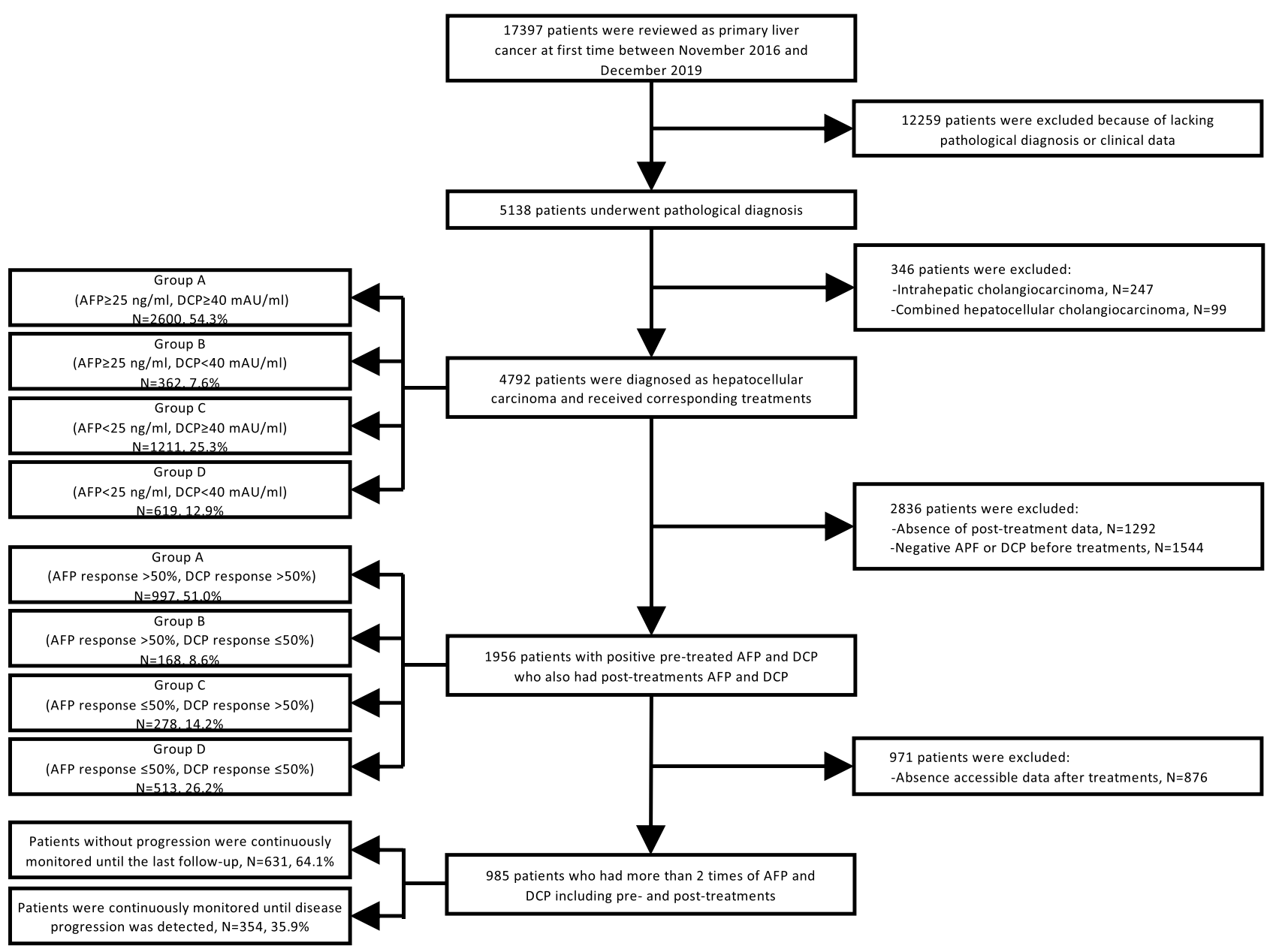

Figure I Flow diagram of the patients who were enrolled in the present analysis and the criteria for grouping.

ascites $(109,5.8 \%)$ among the 4 groups. Interestingly, patients who were AFP positive but DCP negative (group B) presented unique characteristics. When compared to group $\mathrm{C}$, in which patients showed positive DCP but negative AFP, tumours in group B were less aggressive regarding tumour size $(\leq 2.0 \mathrm{~cm}, 29.0 \%$ vs $8.7 \%, P<0.001$ ), tumour number (one tumour, $34.3 \%$ vs $25.8 \%, P<0.001)$ and vascular invasion (presence of vascular invasion, $3.3 \%$ vs $5.9 \%, P<0.001$ ) (Table 1 ).

\section{Initial Analysis of Pretreatment AFP and DCP}

The AFP and DCP levels of patients with different clinical characteristics were compared (Figure 2A-D). Radical therapies included liver resection (LR) and radiofrequency ablation (RFA), and nonradical therapies included transarterial chemoembolization (TACE) and hepatic arterial infusion chemotherapy (HAIC). The AFP and DCP levels were significantly increased in patients who did not receive radical therapies (median [interquartile range], 115.40 [2894.35] vs 32.99 [484.70], $P<0.0001 ; 1109.00$ [7230.00] vs 122.00 [897.50] $P<0.0001$ for AFP and DCP, respectively) or died at the end of follow-up (47.23 [624.85] vs $506.80 \quad[7603.26], \quad P<0.0001 ; \quad 207.50$ [1814.00] vs 24007.00 [11151.00], $P<0.0001$ for AFP and DCP, respectively) compared with patients who received radical therapies or survived at the end of followup. Moreover, AFP and DCP levels were also significantly higher in tumours that were larger than $5 \mathrm{~cm}$ in diameter (34.96 [340.99] vs 32.85 [458.11] vs 156.30 [4678.69], $P<0.0001 ; 43.50$ [129.25] vs 144.00 [652.50] vs 2506.00 [10448.75], $P<0.0001$ for AFP and DCP, respectively) or in the presence of vascular invasion (48.83 [710.69] vs 401.50 [6498.77], $P<0.0001 ; 220.00$ [1950.50] vs 2599.50 [9536.00], $P<0.0001$ for AFP and DCP, respectively) than in tumours that were smaller in the absence of vascular invasion (Figure 2A-D). 
Table I Baseline Characteristics of HCC Cases from 4 Groups

\begin{tabular}{|c|c|c|c|c|c|c|c|}
\hline & & $\begin{array}{l}\text { Group A (2600, } \\
54.3 \%)\end{array}$ & $\begin{array}{l}\text { Group } \\
\text { B (362, 7.6\%) }\end{array}$ & $\begin{array}{l}\text { Group } \\
\text { C (12II, } \\
25.3 \%)\end{array}$ & $\begin{array}{l}\text { Group } \\
\text { D (619, } \\
\text { |2.9\%) }\end{array}$ & $\begin{array}{l}\text { P* } \\
\text { B vs C }\end{array}$ & $\begin{array}{l}P^{\S} \\
\text { A vs } \\
\text { B vs } \\
\text { C vs D }\end{array}$ \\
\hline Age (years) & & $54.94 \pm 11.98$ & $55.38 \pm|2.8|$ & $59.26 \pm 11.68$ & $57.66 \pm 12.04$ & $<0.001$ & $<0.001$ \\
\hline Gender (male/female, \%) & & $\begin{array}{l}2296 / 304(88.3 / \\
11.7)\end{array}$ & $\begin{array}{l}262 / 100(72.4 / \\
27.6)\end{array}$ & $\begin{array}{l}1104 / 107(91.2 / \\
8.8)\end{array}$ & $\begin{array}{l}491 / 128(79.3 / \\
20.7)\end{array}$ & $<0.001$ & $<0.001$ \\
\hline Hepatis B surface antigen $( \pm, \%)$ & Unknown & $\begin{array}{l}1997(76.8) / 363 \\
(14.0) \\
240(9.2)\end{array}$ & $\begin{array}{l}280(86.2) / 45 \\
(12.4) \\
37(10.2)\end{array}$ & $\begin{array}{l}864(79.9) / 218 \\
(18.0) \\
129(10.7)\end{array}$ & $\begin{array}{l}422(74.6) / 144 \\
(23.3) \\
53(8.6)\end{array}$ & 0.037 & $<0.001$ \\
\hline Hepatis C virus antibody ( \pm , \%) & Unknown & $\begin{array}{l}70(2.7) / 2290 \\
(88.1) \\
240(9.2)\end{array}$ & $\begin{array}{l}15(4.1) / 310 \\
(85.6) \\
37(10.2)\end{array}$ & $\begin{array}{l}33(2.7) / 1049 \\
(86.6) \\
129(10.7)\end{array}$ & $\begin{array}{l}\text { II }(1.8) / 555 \\
(89.7) \\
53(8.6)\end{array}$ & 0.383 & 0.431 \\
\hline $\begin{array}{l}\text { Child-Pugh classifications (A/B/ } \\
\text { C, \%) }\end{array}$ & Unknown & $\begin{array}{l}\text { II } 53(44.3) / 36 \\
(1.4) / 0(0.0) \\
1411(54.3)\end{array}$ & $\begin{array}{l}169(46.7) / 6 \\
(1.7) / 0(0.0) \\
187(51.7)\end{array}$ & $\begin{array}{l}604(49.9) / 10 \\
(0.8) / 1(0.0) \\
596(49.2)\end{array}$ & $\begin{array}{l}283(45.7) / 5 \\
(0.8) / 0(0.0) \\
331(53.5)\end{array}$ & 0.285 & 0.511 \\
\hline Platelet count $\left(\times 10^{9} / \mathrm{L}\right)$ & Unknown & $\begin{array}{l}206.83 \pm 102.28 \\
109(4.2)\end{array}$ & $\begin{array}{l}157.09 \pm 69.95 \\
\text { II }(3.0)\end{array}$ & $\begin{array}{l}195.07 \pm 89.24 \\
44(3.6)\end{array}$ & $\begin{array}{l}|78.4| \pm 86.43 \\
15(2.4)\end{array}$ & $<0.001$ & $<0.001$ \\
\hline Albumin (mg/dL) & Unknown & $\begin{array}{l}40.88 \pm 4.80 \\
122(4.7)\end{array}$ & $\begin{array}{l}41.84 \pm 4.75 \\
17(4.7)\end{array}$ & $\begin{array}{l}41.50 \pm 4.56 \\
51(4.2)\end{array}$ & $\begin{array}{l}41.87 \pm 4.54 \\
16(2.6)\end{array}$ & 0.230 & $<0.001$ \\
\hline Alanine aminotransferase (U/L) & Unknown & $\begin{array}{l}51.01 \pm 49.32 \\
122(4.7)\end{array}$ & $\begin{array}{l}37.81 \pm 30.30 \\
16(4.4)\end{array}$ & $\begin{array}{l}45.08 \pm 46.41 \\
51(4.2)\end{array}$ & $\begin{array}{l}35.51 \pm 25.25 \\
17(2.7)\end{array}$ & 0.006 & $<0.001$ \\
\hline Aspartate aminotransferase (U/L) & Unknown & $\begin{array}{l}67.47 \pm 70.30 \\
122(4.7)\end{array}$ & $\begin{array}{l}37.86 \pm 28.43 \\
16(4.4)\end{array}$ & $\begin{array}{l}46.63 \pm 51.21 \\
51(4.2)\end{array}$ & $\begin{array}{l}33.48 \pm 21.20 \\
17(2.7)\end{array}$ & 0.002 & $<0.001$ \\
\hline Total bilirubin (umol/L) & Unknown & $\begin{array}{l}19.20 \pm 25.49 \\
122(4.7)\end{array}$ & $\begin{array}{l}15.55 \pm 9.19 \\
17(4.7)\end{array}$ & $\begin{array}{l}\mid 7.50 \pm 31.53 \\
5 \mid(4.2)\end{array}$ & $\begin{array}{l}14.54 \pm 8.00 \\
16(2.6)\end{array}$ & 0.256 & $<0.001$ \\
\hline Prothrombin time (s) & Unknown & $\begin{array}{l}12.21 \pm 1.17 \\
1139(43.8)\end{array}$ & $\begin{array}{l}12.32 \pm 1.17 \\
14 \mid(39.0)\end{array}$ & $\begin{array}{l}12.00 \pm 1.20 \\
484(40.0)\end{array}$ & $\begin{array}{l}12.2 \mid \pm 1.55 \\
260(42.0)\end{array}$ & $<0.001$ & $<0.001$ \\
\hline $\begin{array}{l}\text { Activated partial } \\
\text { thromboplastin time (s) }\end{array}$ & Unknown & $\begin{array}{l}27.93 \pm 4.59 \\
1139(43.8)\end{array}$ & $\begin{array}{l}28.93 \pm 5.08 \\
|4|(39.0)\end{array}$ & $\begin{array}{l}27.17 \pm 4.30 \\
484(40.0)\end{array}$ & $\begin{array}{l}28.40 \pm 5.44 \\
260(42.0)\end{array}$ & 0.004 & 0.005 \\
\hline $\begin{array}{l}\text { Tumor numbers (one/two/more } \\
\text { than two, \%) }\end{array}$ & Unknown & $\begin{array}{l}493(19.0) / 202 \\
(7.8) / 1001(38.5) \\
904(34.8)\end{array}$ & $\begin{array}{l}124(34.3) / 27 \\
(7.5) / 95(26.2) \\
116(32.0)\end{array}$ & $\begin{array}{l}313(25.8) / 123 \\
(10.2) / 397(32.8) \\
378(31.2)\end{array}$ & $\begin{array}{l}164(26.5) / 52 \\
(8.4) / I 58(25.5) \\
245(39.6)\end{array}$ & 0.004 & $<0.001$ \\
\hline $\begin{array}{l}\text { Tumor size }(\leq 2.0 \mathrm{~cm} / 2.1- \\
5.0 \mathrm{~cm} />5.0 \mathrm{~cm}, \%)\end{array}$ & Unknown & $\begin{array}{l}194(7.5) / 521 \\
(20.0) / 884(33.4) \\
1001(38.5)\end{array}$ & $\begin{array}{l}105(29.0) / 105 \\
(29.0) / 27(7.5) \\
125(34.5)\end{array}$ & $\begin{array}{l}105(8.7) / 366 \\
(30.2) / 338(27.9) \\
402(33.2)\end{array}$ & $\begin{array}{l}140(22.6) / 177 \\
(28.6) / 48(7.8) \\
254(4 I .0)\end{array}$ & $<0.001$ & $<0.001$ \\
\hline Presence vascular invasion $( \pm, \%)$ & Unknown & $\begin{array}{l}330(12.7) / 1560 \\
(60.0) \\
710(27.3)\end{array}$ & $\begin{array}{l}12(3.3) / 265 \\
(73.2) \\
85(23.5)\end{array}$ & $\begin{array}{l}71(5.9) / 856 \\
(70.7) \\
284(23.5)\end{array}$ & $\begin{array}{l}10(1.6) / 424 \\
(68.5) \\
185(29.9)\end{array}$ & 0.159 & $<0.001$ \\
\hline $\begin{array}{l}\text { Presence extrahepatic } \\
\text { metastasis }( \pm, \%)\end{array}$ & Unknown & $\begin{array}{l}272(10.5) / 1533 \\
(59.0) \\
795(30.6)\end{array}$ & $\begin{array}{l}24(6.6) / 238 \\
(65.7) \\
100(27.6)\end{array}$ & $\begin{array}{l}96(10.8) / 795 \\
(65.6) \\
320(26.4)\end{array}$ & $\begin{array}{l}37(9.1) / 370 \\
(59.8) \\
212(34.2)\end{array}$ & 0.682 & $<0.001$ \\
\hline
\end{tabular}


Table I (Continued).

\begin{tabular}{|c|c|c|c|c|c|c|c|}
\hline & & $\begin{array}{l}\text { Group A (2600, } \\
54.3 \%)\end{array}$ & $\begin{array}{l}\text { Group } \\
\text { B (362, 7.6\%) }\end{array}$ & $\begin{array}{l}\text { Group } \\
\text { C (121 I, } \\
25.3 \%)\end{array}$ & $\begin{array}{l}\text { Group } \\
\text { D (619, } \\
\text { I2.9\%) }\end{array}$ & $\begin{array}{l}P^{*} \\
\text { B vs C }\end{array}$ & $\begin{array}{l}P^{\S} \\
\text { A vs } \\
\text { B vs } \\
\text { C vs D }\end{array}$ \\
\hline $\begin{array}{l}\text { Barcelona Clinic Liver Cancer } \\
\text { staging }(A / B / C, \%)\end{array}$ & Unknown & $\begin{array}{l}515(19.8) / 716 \\
(27.5) / 502(19.3) \\
867(33.3)\end{array}$ & $\begin{array}{l}167(46.1) / 47 \\
(13.0) / 32(8.8) \\
116(32.0)\end{array}$ & $\begin{array}{l}340(28.1) / 356 \\
(29.4) / 147(12.1) \\
368(30.4)\end{array}$ & $\begin{array}{l}218(35.2) / 116 \\
(18.7) / 44(7.1) \\
241(38.9)\end{array}$ & $<0.001$ & $<0.001$ \\
\hline Presence of ascites $( \pm, \%)$ & Unknown & $\begin{array}{l}109(4.2) / 1781 \\
(68.5) \\
710(27.3)\end{array}$ & $\begin{array}{l}11(3.0) / 266 \\
(73.5) \\
85(23.5)\end{array}$ & $\begin{array}{l}27(2.2) / 900 \\
(74.3) \\
284(23.5)\end{array}$ & $\begin{array}{l}17(2.7) / 417 \\
(67.4) \\
185(29.9)\end{array}$ & 0.676 & 0.002 \\
\hline $\begin{array}{l}\text { Tumor differentiations (well/ } \\
\text { well-moderate/moderate/ } \\
\text { moderate poor/poor, \%) }\end{array}$ & Unknown & $\begin{array}{l}19(0.7) / 16(0.6) / \\
413(15.9) / 399 \\
(15.3) / 176(6.8) \\
1577(60.7)\end{array}$ & $\begin{array}{l}2(0.6) / 6(1.7) / \\
61(16.9) / 45 \\
(12.4) / 19(5.2) \\
229(63.3)\end{array}$ & $\begin{array}{l}37(3.1) / 45(3.7) / \\
315(26.0) / 118 \\
(9.7) / 37(3.1) \\
659(54.4)\end{array}$ & $\begin{array}{l}22(3.6) / 18 \\
(2.9) / 86(13.9) / \\
27(4.4) / 23(3.7) \\
443(71.6)\end{array}$ & $<0.001$ & $<0.001$ \\
\hline $\begin{array}{l}\text { Presence microvascular } \\
\text { invasion }(0 / 1 / 2, \%)\end{array}$ & Unknown & $\begin{array}{l}12(4.7) / 54(2.1) / \\
19(0.7) \\
2404(92.5)\end{array}$ & $\begin{array}{l}24(6.6) / 7(1.9) / \\
0(0.0) \\
331(91.4)\end{array}$ & $\begin{array}{l}93(7.7) / 23(1.9) / \\
4(0.3) \\
1091(90.1)\end{array}$ & $\begin{array}{l}I 8(2.9) / /(0.2) / \\
0(0.0) \\
600(96.9)\end{array}$ & 0.644 & $<0.001$ \\
\hline $\begin{array}{l}\text { Treatments (LR/RFA/TACE/ } \\
\text { HAIC, \%) }\end{array}$ & & $\begin{array}{l}711(27.3) / 313 \\
(12.1) / 626(24.1) / \\
950(36.5)\end{array}$ & $\begin{array}{l}120(33.1) / 144 \\
(39.8) / 54 \\
(14.9) / 44(12.2)\end{array}$ & $\begin{array}{l}464(38.3) / 220 \\
(18.2) / 252 \\
(20.8) / 275(22.7)\end{array}$ & $\begin{array}{l}194(31.3) / 254 \\
(4 I . I) / 89(14.4) / \\
82(13.2)\end{array}$ & $<0.001$ & $<0.001$ \\
\hline $\begin{array}{l}\text { Progression-free survival } \\
\text { (months) }\end{array}$ & & $5.66 \pm 6.89$ & $8.74 \pm 8.72$ & $7.78 \pm 8.05$ & $8.22 \pm 8.81$ & 0.009 & $<0.001$ \\
\hline Overall survival (months) & & $10.25 \pm 10.90$ & $14.45 \pm 12.94$ & $12.82 \pm 11.89$ & $12.62 \pm 12.32$ & $<0.001$ & $<0.001$ \\
\hline
\end{tabular}

Notes: Data are presented as mean \pm standard deviations or numbers with percentages in parentheses. Patients were divided into 4 groups, Group $A$ (AFP $\geq 25 \mathrm{ng} / \mathrm{mL}$, $\mathrm{DCP} \geq 40 \mathrm{mAU} / \mathrm{mL})$, Group B (AFP $\geq 25 \mathrm{ng} / \mathrm{mL}, \mathrm{DCP}<40 \mathrm{mAU} / \mathrm{mL})$, Group C (AFP<25 ng/mL, DCP $\geq 40 \mathrm{mAU} / \mathrm{mL}), \mathrm{Group} \mathrm{D}(A F P<25 \mathrm{ng} / \mathrm{mL}, \mathrm{DCP}<40 \mathrm{mAU} / \mathrm{mL})$. ${ }^{*}$ Comparisons of difference between Group B and C. ${ }^{\S}$ Comparisons of difference among four groups.

Abbreviations: HCC, hepatocellular carcinoma; AFP, alpha-fetoprotein; DCP, des-gamma-carboxy prothrombin; LR, liver resection; RFA, radiofrequency ablation; TACE, transarterial chemoembolization; HAIC, hepatic arterial infusion chemotherapy.

In the survival analysis, the median PFS times for groups A, B, C and D were 6.067 (95\% confidence interval $[95 \% \mathrm{CI}], 5.533-6.567$ months), 18.767 (95\% CI, 15.567-28.967 months), 13.633 (95\% CI, 11.700-15.800 months) and 28.833 (95\% CI, 22.867-43.833 months) months, respectively. Patients in group A who were both AFP- and DCP-positive suffered from the lowest 1-, 2and 3-year PFS rates, with $34.3 \%, 22.3 \%$ and $16.9 \%$, respectively, compared to the highest group $\mathrm{D}$, with $65.2 \%, 53.1 \%$ and $44.9 \%(P<0.0001)$. Groups $\mathrm{B}$ and $\mathrm{C}$ stood at medium positions, of which group $\mathrm{B}$ was significantly better than group $\mathrm{C}$ in terms of the 1-, 2- and 3 -year PFS rates, which were $59.7 \%, 44.7 \%$ and $36.9 \%$ and $52.8 \%, 35.7 \%$ and $28.1 \%$, respectively $(P=0.009$, Figure 2E). Regarding OS, although there were no groups that reached a median OS time point, patients in group A who were AFP and DCP positive had the lowest 1-, 2- and 3-year OS rates with $76.3 \%, 72.2 \%$ and $69.4 \%$, respectively, compared to group B with $94.0 \%, 91.9 \%$ and $91.9 \% \quad(P<0.0001)$, respectively, group $\mathrm{C}$ with $88.2 \%, 83.4 \%$ and $81.7 \%(P<0.0001)$, respectively, and that of group $\mathrm{D}$ with $94.0 \%, 91.8 \%$ and $89.7 \%$ $(P<0.0001$, Figure $2 \mathrm{~F})$, respectively. The median followup times of 4792 patients were 4.03 months and 7.25 months for PFS and OS, respectively. The PFS and OS of the entire population are shown in the supplementary materials (Figure S1b and S1c).

\section{Response Analysis of Posttreatment AFP and DCP}

A total of 1956 patients who were positive for AFP and DCP before treatments and whose tumour markers were monitored the first month after treatment were evaluated for AFP and DCP response (Figure 3). Generally, the PFS 
A
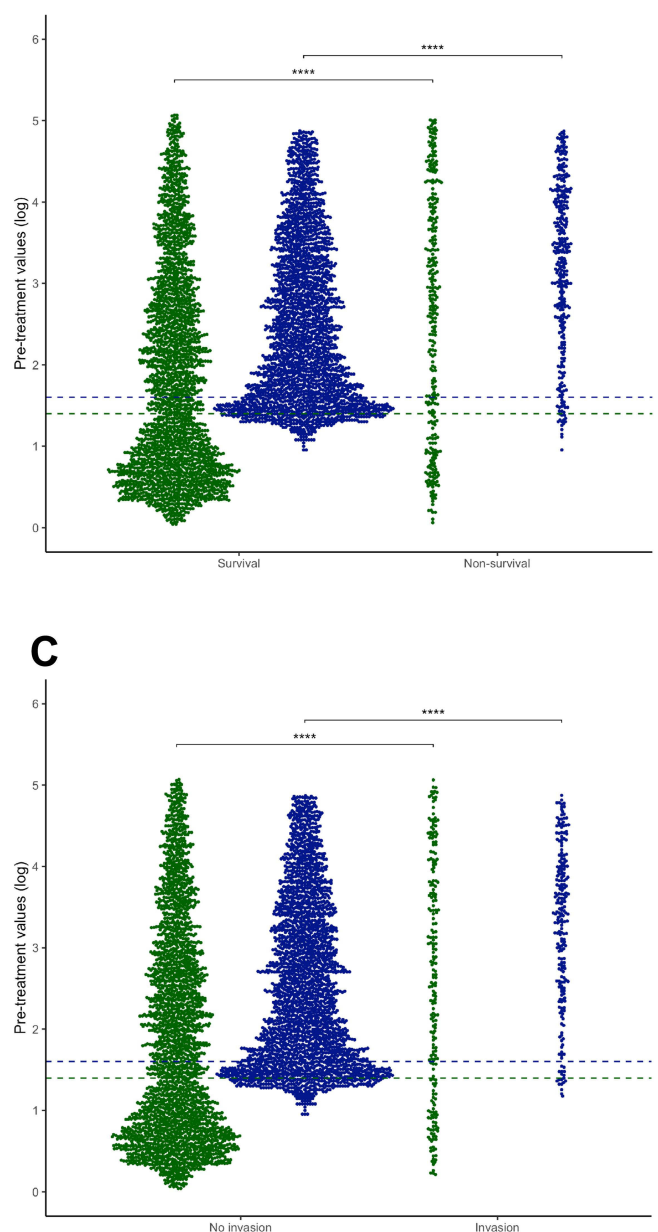

E

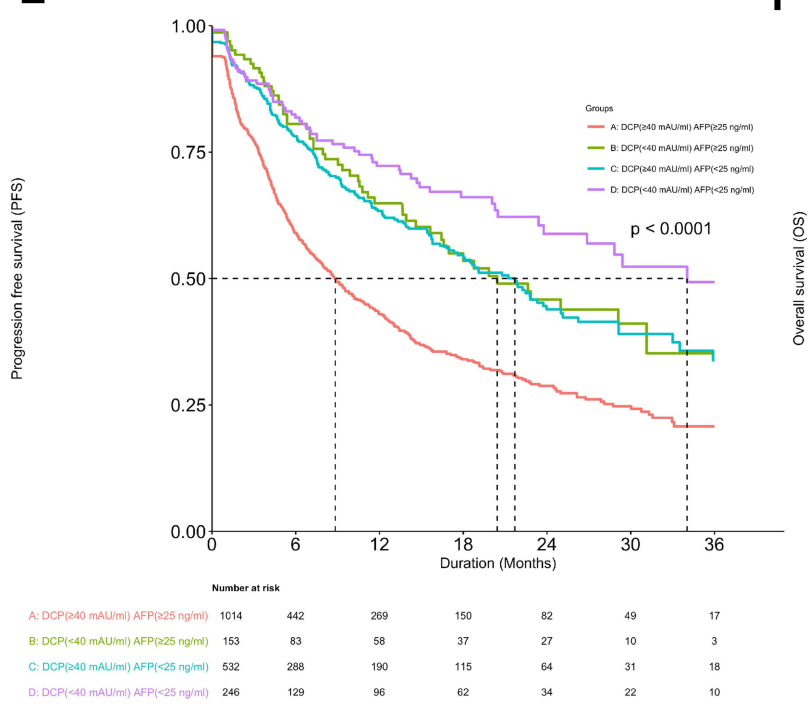

B

D

F
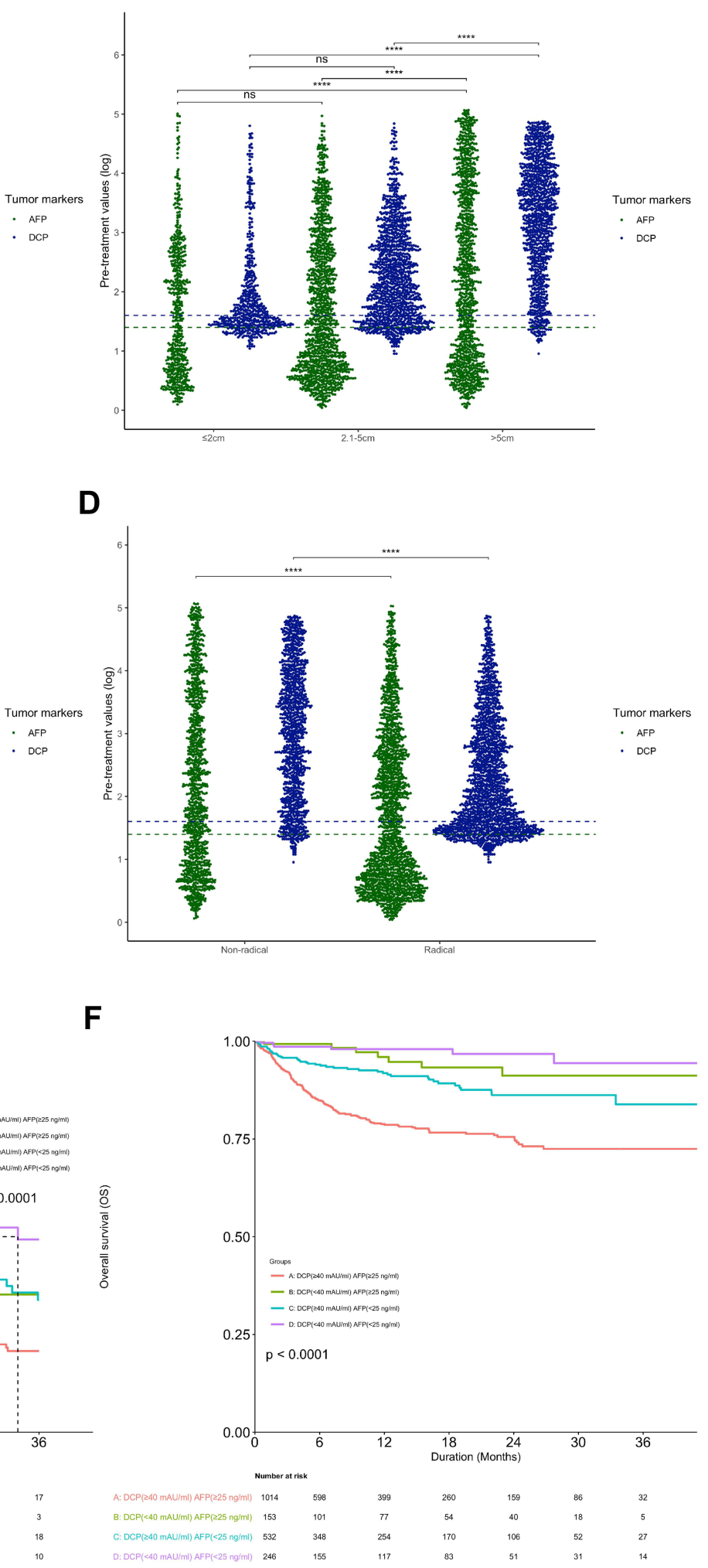

Figure 2 Log-transformed alpha-fetoprotein (AFP) and des-gamma-carboxyprothrombin (DCP) levels subdivided according to survival status (A), tumour sizes (B), presence of vascular invasion (C), and treatment models (D). Progression-free survival (PFS) (E) and overall survival (OS) (F) curves of grouped hepatocellular carcinoma patients based on pretreatment AFP and DCP. Reference dashed lines were drawn for diagnostic cut-off values for AFP [log(25)] and DCP [log(40)] in corresponding colours (A-D). Group A, AFP $\geq 25 \mathrm{ng} / \mathrm{mL}$, DCP $\geq 40 \mathrm{mAU} / \mathrm{mL}$; Group B, AFP $\geq 25 \mathrm{ng} / \mathrm{mL}$, DCP $<40 \mathrm{mAU} / \mathrm{mL}$; Group C, AFP $<25 \mathrm{ng} / \mathrm{mL}$, DCP $\geq 40 \mathrm{mAU} / \mathrm{mL}$; Group D, AFP $<25 \mathrm{ng} / \mathrm{mL}$, $\mathrm{DCP}<40 \mathrm{mAU} / \mathrm{mL}$, (E and $\mathbf{F})$. ${ }^{*} * * * P<0.000$ I (Wilcoxon rank-sum test). 
A

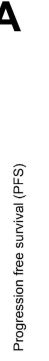
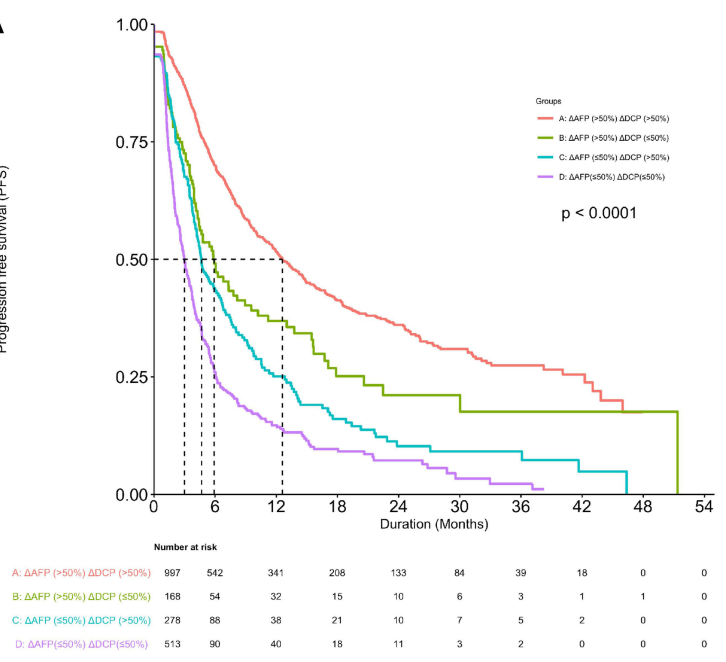

C

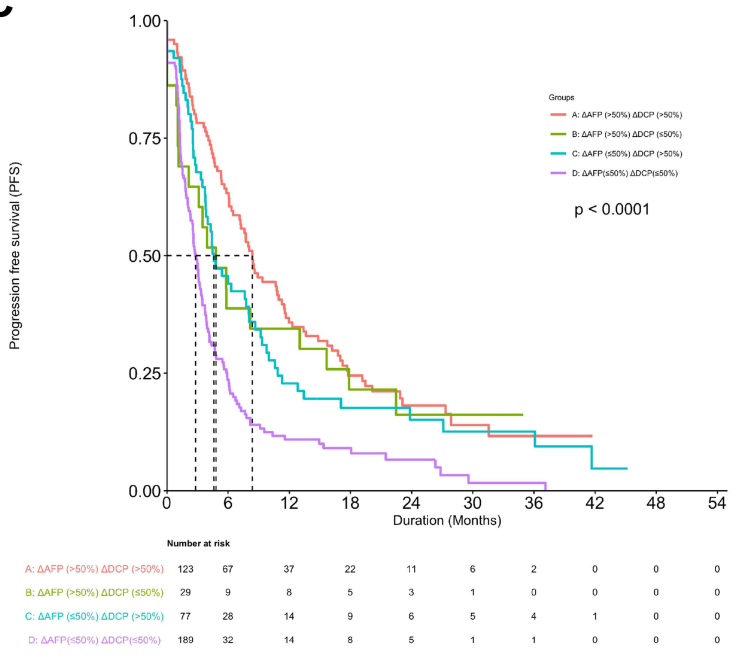

E

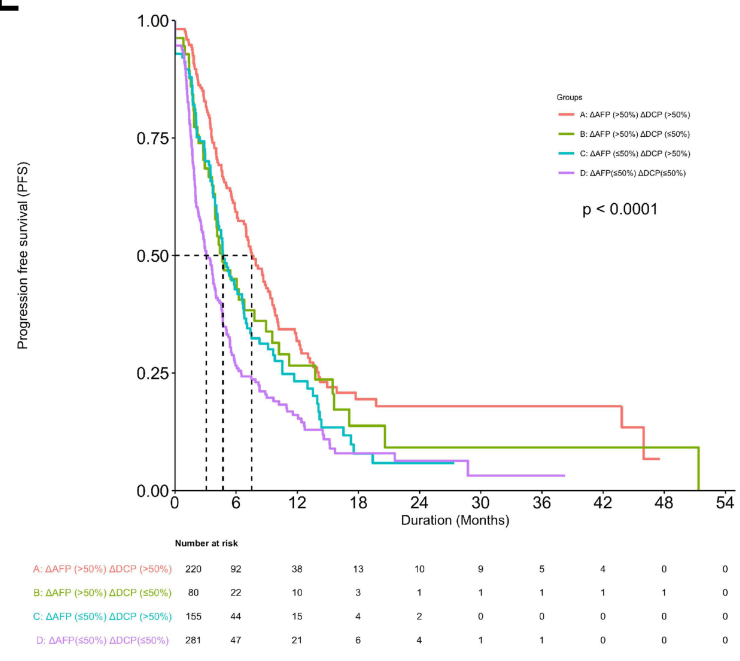

B

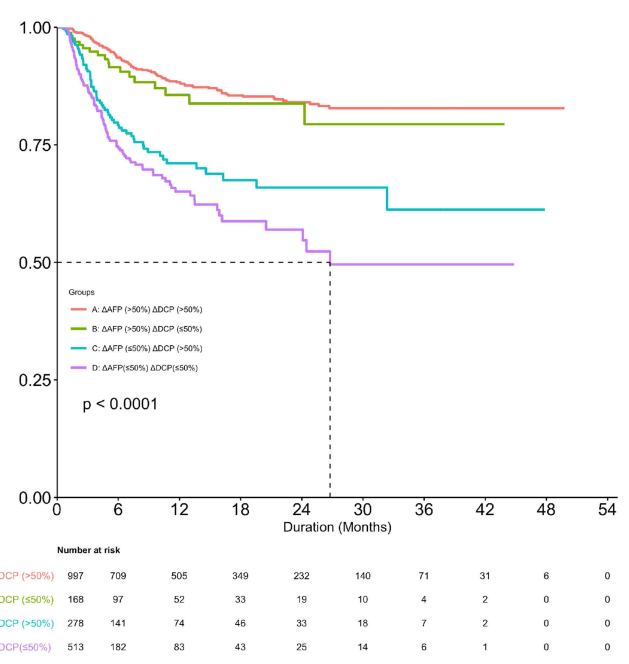

D

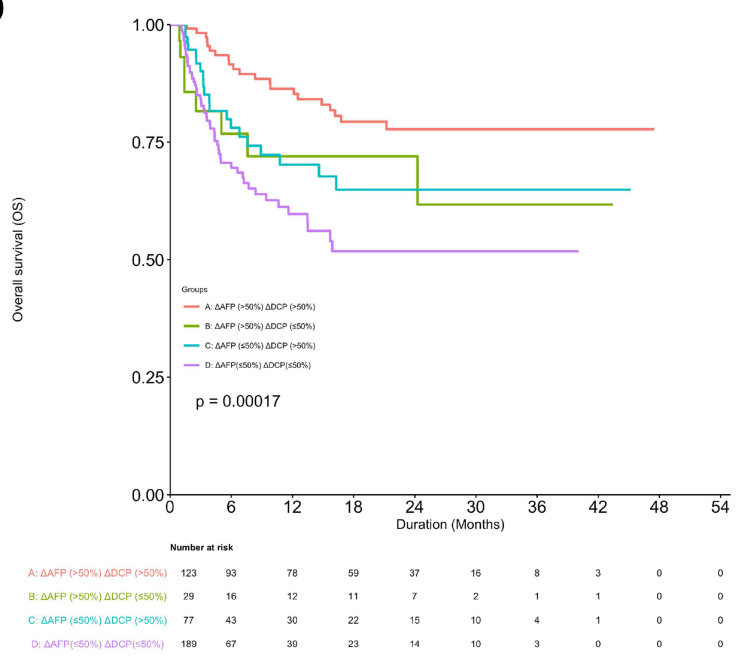

F

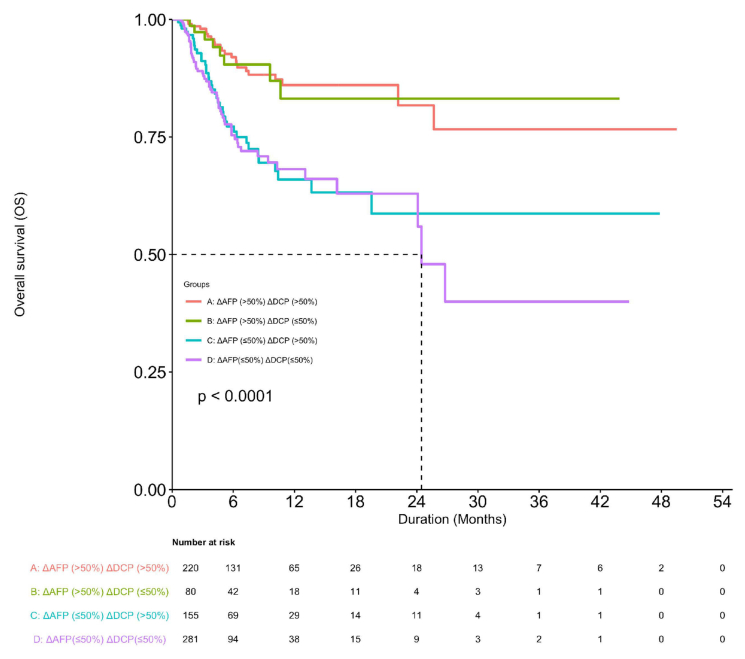

Figure 3 Progression-free survival (PFS) (A, C, E) and overall survival (OS) (B, D, F) curves of grouped hepatocellular carcinoma patients based on the alpha-fetoprotein (AFP) and des-gamma-carboxyprothrombin (DCP) response rates after all treatments (A and $\mathbf{B})$, transarterial chemoembolization (C and $\mathbf{D}$ ), and hepatic arterial infusion chemotherapy (E and $\mathbf{F}$ ). Group A, AFP response $>50 \%$, DCP response $>50 \%$; Group B, AFP response $>50 \%$, DCP response $\leq 50 \%$; Group C, AFP response $\leq 50 \%$, DCP response $>50 \%$; Group D, AFP response $\leq 50 \%$, DCP response $\leq 50 \%$. 
and OS of the 1956 patients indicated that both AFP and DCP responses less than $50 \%$ after initial treatments represented significantly worse survival outcomes $(P<0.0001$, Figure $3 \mathrm{~A}$ and B). More specifically, patients whose AFP and DCP did not decrease by more than $50 \%$ after the first TACE or HAIC demonstrated significantly shorter PFS $(P<0.0001)$ and $\mathrm{OS}(P=0.0002, P<0.0001$; Figure $3 \mathrm{C}-\mathrm{F})$. Correspondingly, good AFP and DCP responses that declined more than $50 \%$ compared to pretreatment presented the best survival benefits. Moreover, a single AFP response greater than $50 \%$ demonstrated better OS for patients after HAIC $(P<0.0001$; Figure $3 F)$. The AFP and DCP responses regarding radical-intended treatment, including LR and RFA, are depicted in the supplementary materials (Figure S2a-d). Briefly, 91.9\% of patients had reduced AFP and DCP by more than $50 \%$. AFP and DCP responses less than $50 \%$ were associated with significantly lower recurrence-free survival (RFS) times for both LR $(P=0.0015)$ and RFA ( $P=0.0029$; Figure S2a and c) . Neither AFP nor DCP responses less than 50\% after RFA indicated significantly worse OS than other groups ( $P=0.0100)$, but there was no difference for patients who underwent LR regarding OS $(P=0.7700)$.

\section{Long-Term Follow-Up Analysis of AFP and DCP After Treatment}

There were 885 patients who underwent radical treatment and whose AFP and DCP levels were continuously monitored after treatment. A total of 631 versus 354 patients were progression-free or showed disease progression by the last follow-up, respectively (Figure 1). Generally, both AFP and DCP demonstrated flat trends under the diagnostic threshold for the majority of progression-free patients during long-term surveillance. Moreover, DCP demonstrated a more sensitive response than AFP, returning to a normal value, while AFP required a longer time to recover to normal after the initial treatments (Figure $4 \mathrm{~A}$ and B). The line charts for those patients who had disease progression detected at the last follow-up are depicted in the supplementary materials (Figure S3a and b).

\section{Prognostic Factors for Recurrence-Free Survival and Overall Survival}

Factors associated with PFS and OS are reported in Table $\underline{\mathrm{S}}$. Univariate analysis identified the following significant variables for both PFS and OS: sex, presence of ascites, tumour number, tumour size, presence of vascular invasion, presence of extrahepatic metastasis, and pretreatment levels of ALB, TBIL, APTT, AFP and DCP $(P<0.010)$.

Patients with complete research-interested clinical data based on univariate analysis were enrolled in the multivariate regression model for analysis; as a result, 1945 patients remained for the following analysis. According to multivariate analysis, male (hazard ratio [HR], 1.39; 95\% CI, 1.1-1.7; $P=0.005$ ), lower ALB (HR, 1.65; 95\% CI, 1.2-2.2; $P<0.001$ ), presence of ascites (HR, 1.68; 95\% CI, 1.1-2.5; $P=0.008)$, longer APTT (HR, 0.73; 95\% CI, $0.6-0.9 ; P=0.003)$, more than two tumours (HR, 1.78; 95\% CI, 1.5-2.1; $P<0.001)$, tumour larger than $5 \mathrm{~cm}$ (HR, 1.43; 95\% CI, 1.1-1.8; $P=0.002$ ), presence of vascular invasion (HR, 1.75; 95\% CI, 1.4-2.2; $P<0.001$ ), presence of extrahepatic metastasis (HR, 1.70; 95\% CI, 1.4-2.1; $P<0.001)$, and positive pretreatment AFP (HR, 1.57; 95\% CI, 1.3$1.8 ; P<0.001)$ and DCP (HR, 1.25; 95\% CI, 1.0-1.6; $P=0.05)$ were identified as independent factors for worse PFS (Figure 5). Regarding OS, lower ALB (HR, 2.04; 95\% CI, 1.3-3.2; $P=0.003$ ), presence of ascites (HR, $1.98 ; 95 \% \mathrm{CI}, 1.2-3.3 ; P=0.009)$, more than two tumours (HR, 1.71; 95\% CI, 1.3-2.3; $P<0.001$ ), tumour larger than $5 \mathrm{~cm}$ (HR, 2.35; 95\% CI, 1.5-3.8; $P<0.001$ ), presence of vascular invasion ( $\mathrm{HR}, 2.06 ; 95 \% \mathrm{CI}, 1.5-2.9 ; P<0.001)$, presence of extrahepatic metastasis (HR, 1.87; 95\% CI, 1.4-2.6; $P<0.001$ ), and positive pretreatment AFP (HR, $1.81 ; 95 \% \mathrm{CI}, 1.3-2.5 ; P<0.001)$ and DCP (HR, 2.14; 95\% CI, 1.2-3.9; $P=0.012$ ) were most significant predictors (Figure S4). Additionally, the PFS and OS curves of 1945 patients regarding different groups are presented in the supplementary materials, which indicated similar distributions to the 4792-general group (Figure S1d and e).

\section{Discussion}

In the current study, we explored the values of two routine clinical tumour biomarkers for patients with HCC, AFP and DCP, regarding prognosis predicting and recurrence monitoring. Acquisition of reliable biological behaviour information from tumours is of clinical importance for defining treatment strategies in patients with HCC. Although AFP has been recommended by HCC guidelines for many years, the sensitivity is not satisfactory. ${ }^{13}$ In our study, only $61.8 \%$ of patients revealed AFP positivity at their initial visit to the hospital, whereas the sensitivity of DCP reached $79.5 \%$. A total of 1211/4792 (25.3\%) patients were DCP positive but AFP negative, accounting for a quarter of the entire HCC population in this study. In addition, when carefully 

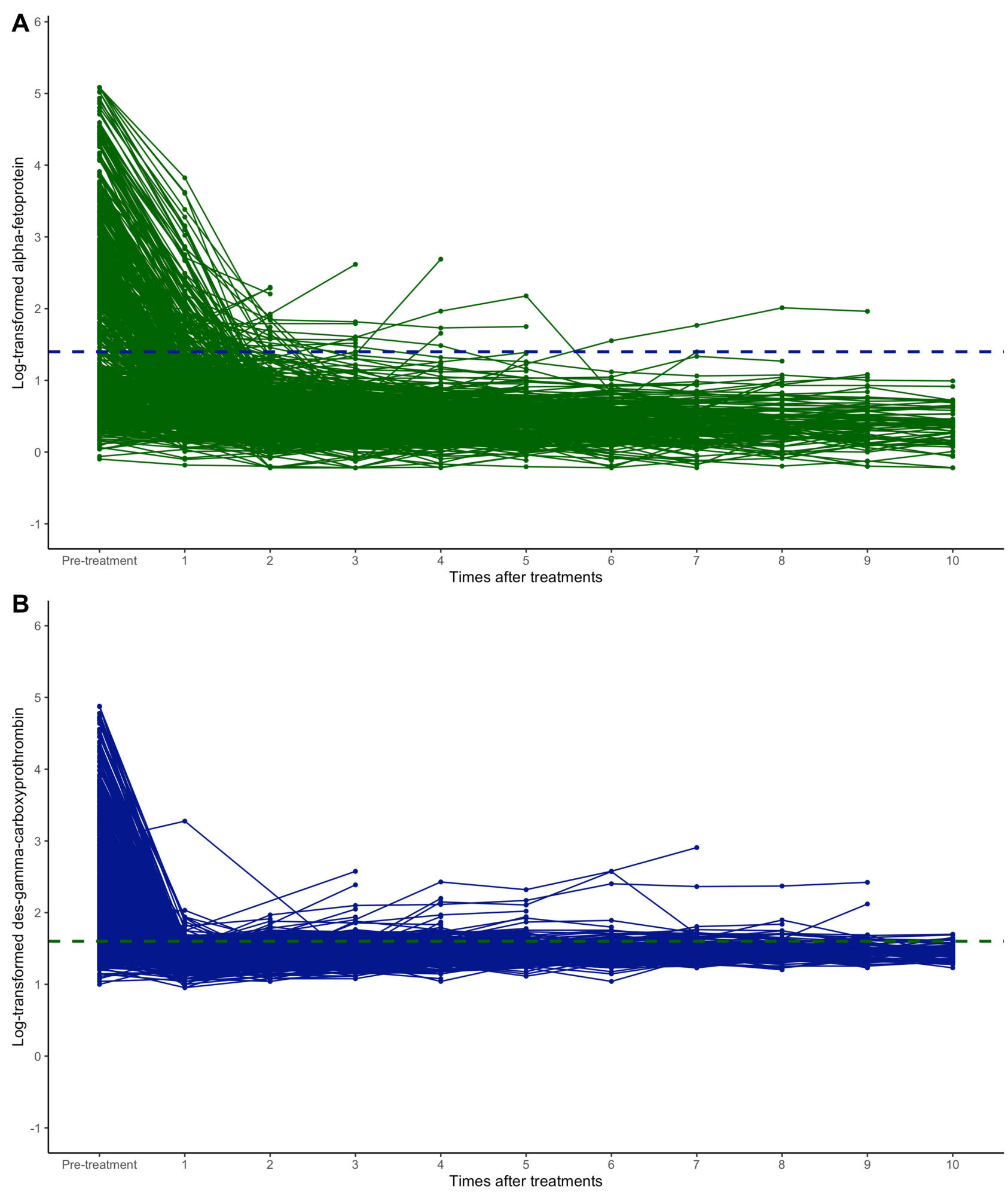

Figure 4 Log-transformed alpha-fetoprotein (AFP) (A) and des-gamma-carboxyprothrombin (DCP) (B) at each follow-up after the first treatment among patients who were progression-free. Reference dashed lines were drawn for diagnostic cut-off values for AFP $[\log (25)]$ and DCP $[\log (40)]$ in corresponding plots $(\mathbf{A}$ and $\mathbf{B})$, respectively.

examining the remaining $38.2 \%$ of patients who had negative AFP results, 1211/1830 (66.2\%) of them had positive DCP results, providing further information for these populations that lacked positive predictors. This finding emphasized the necessity of combining AFP and DCP for the surveillance of HCC patients. 


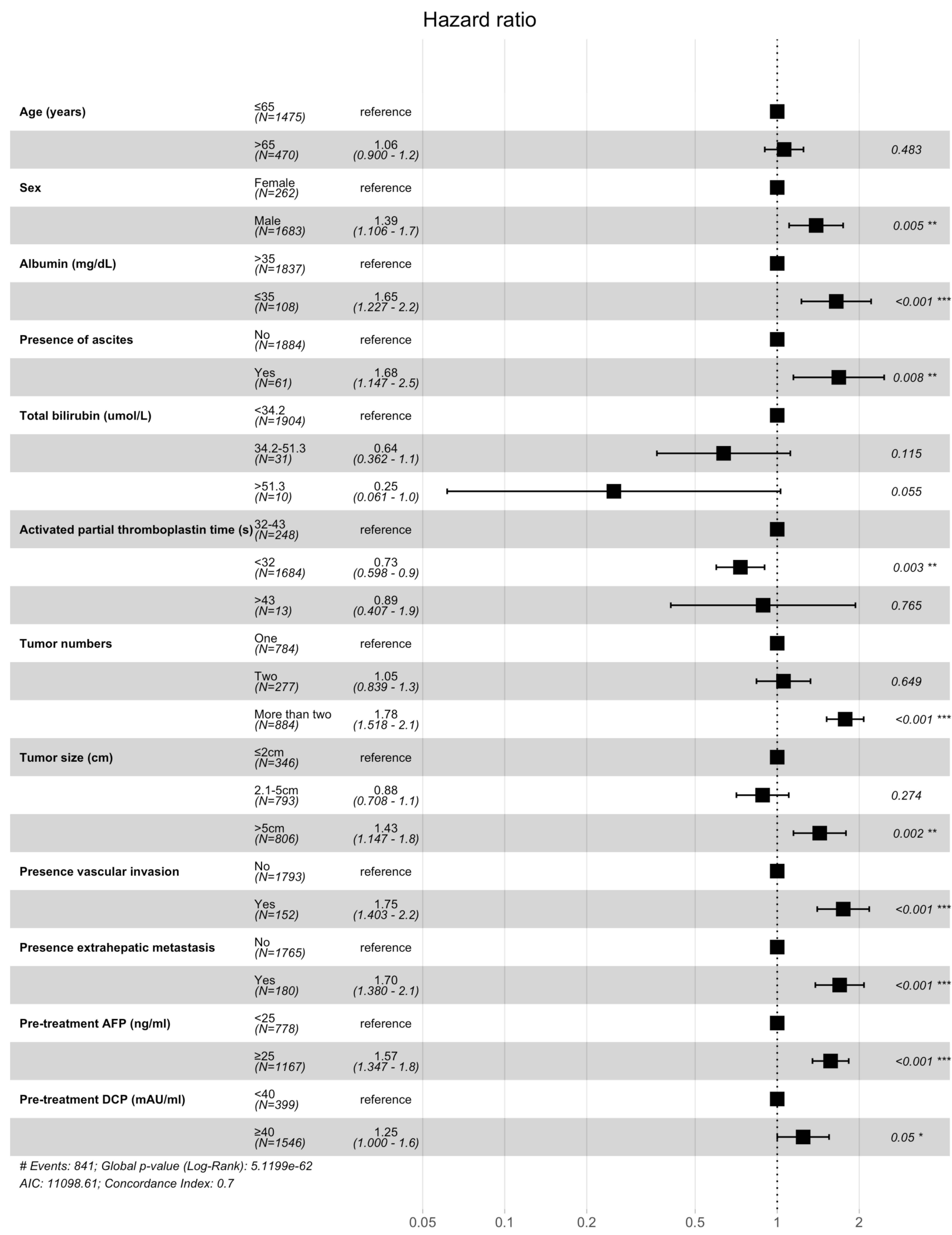

Figure 5 Forest plots from multivariate Cox regression models of progression-free survival. ${ }^{*} P<0.05,{ }^{* *} P<0.01,{ }^{* * * k} P<0.001$.

The combination of AFP and DCP could distinguish tumours with various biological behaviours, facilitating prognostic prediction. Previous studies demonstrated that using tumour markers could predict the degree of HCC malignancy. ${ }^{14}$ Many studies prefer to employ multiple tumour markers and investigate different combinations of 
these markers for diagnosis or surveillance. Studies from Japan tended to apply three tumour markers, AFP, DCP and Lens culinaris agglutinin-reactive fraction of AFP (AFP-L3), for screening, evaluating therapeutic effects and conducting follow-up. ${ }^{15-17}$ However, some scholars from Japan and Taiwan questioned the contribution of AFP-L3 in the diagnostic and prognostic value. ${ }^{15,18}$ Therefore, using a combination of AFP and DCP is thought to be effective in predicting prognosis and monitoring patients with HCC in the present study.

Regarding the current study, patients with shorter survival times, larger tumours, the presence of vascular invasion, or failure to receive radical therapies tended to present higher levels of AFP and DCP than those who did not have the above characteristics. These AFP and DCP distributions were similar to the study from Yang et al regarding tumour characteristics. ${ }^{19}$ Further analysis indicated that patients with the best oncological data were those with double-negative AFP and DCP results, while patients who were double-positive for AFP and DCP had the shortest PFS and OS. As a cross validation, those double-positive patients were characterized by a higher frequency of more than two tumours, larger tumour size and more vascular invasion, suggesting that positive AFP and DCP results reflect aggressive tumour profiles. These data were consistent with previous studies from Japan. ${ }^{15,16,20}$ Therefore, compared to that of other groups, there was a decreased proportion of double-positive patients who were candidates for radical treatments. Furthermore, postoperative pathology indicated higher proportions of poor tumour differentiation and microvascular invasion (MVI). As a result, these double-positive patients who were unlikely to reach ideal PFS or OS should be considered for an early transplant waitlist or receive other intensive therapies perioperatively, such as RFA combined with TACE. ${ }^{21}$

When taking into consideration AFP and DCP the first month after treatments in the response analysis, patients who were double positive for AFP and DCP before treatments provided more information for predicting prognosis. In the subgroup analysis, regarding two interventional therapies, TACE and HAIC, less than 50\% responses of both AFP and DCP after treatments unfortunately predicted a worse prognosis, and these results were consistent with previous studies that used either marker separately. ${ }^{22,23}$ HCC patients who received RFA showed a similar tendency to receive TACE and HAIC. Moreover, the single AFP response of less than $50 \%$ was more than enough to represent shorter RFS compared to the counterparts, which had not been reported previously. Finally, AFP and DCP responses were not recommended for patients with $\mathrm{HCC}$ who underwent LR because the tumour biomarkers of most HCC patients returned to normal in the first month after radical LR. An earlier time point of monitoring should be selected after LR for future study. ${ }^{10,19}$

In the current study, we reported 2 typical patterns of HBV-derived HCC patients with distinctive survival and prognostic outcomes by adopting pre- and posttreatment AFP and DCP as tumour markers, which was consistent with other studies. ${ }^{22-26}$ To further evaluate the values of AFP and DCP, a subgroup of patients who underwent continuous surveillance of AFP and DCP before and after treatments were plotted to inspect the dynamic changes during long-term follow-up. Notably, DCP showed a more significant and rapid response to treatments than AFP, which required a longer time to decrease to normal, indicating that DCP might be a sensitive tumour marker to measure treatment responses, and AFP could predict prognosis. Additionally, the fixed diagnostic values for AFP and DCP were not suitable for dynamic surveillance, as there were patients who did not have disease progression detected during follow-up; however, their amounts of either AFP or DCP were always above the level of diagnosis (Figure 4A and B). Further analysis should thus concentrate on developing a model that gives dynamic thresholds regarding different intervals of follow-up after treatments.

Multivariate analysis using the Cox proportional hazards model demonstrated that AFP and DCP were both independent factors contributing to PFS and OS. Notably, adding DCP to the profile classified a subpopulation from AFPpositive patients, which was a unique subgroup distinct from the double-positive group. Patients with singlepositive AFP had the smallest tumour size, lowest tumour number, second lowest vascular invasion (slightly more than the double-negative group), and the lowest extrahepatic invasion among the 4 groups. These characteristics supported radical treatments and indicated decent PFS and OS. Additionally, this result was consistent with a previous study from Hamamura, although they did not reach significance because of the small case number. ${ }^{20}$

Detecting tumour markers reveals the underlying biological behaviours of different tumours and provides potential guidance for clinical practice. For example, ramucirumab showed improved OS compared to placebo 
in advanced HCC patients with AFP concentrations of more than $400 \mathrm{ng} / \mathrm{mL}$ who had previously been treated with sorafenib. ${ }^{27}$ We surmised that patients with AFPand DCP-positive or less than $50 \%$ response rates may benefit from more intensive treatments because of their invasiveness and could be counseled to receive more high-end imaging examinations and closer follow-up. Conversely, the follow-up period for low-risk patients should refer to the clinical guidelines. ${ }^{2}$ In addition, this study suggested a design for future clinical trials to consider tumour markers in their inclusion or exclusion criteria for neoadjuvant or adjuvant therapies.

There was one major limitation of this study. Due to the retrospective nature of the study, there were missing data regarding demography and histology for some patients, which would not truly reflect the results of the Cox regression analysis. Therefore, these results should be interpreted cautiously, and further prospective studies are warranted. Moreover, the histology of nonalcoholic fatty liver disease, which is another increasingly important aetiology of HCC other than virus-related HCC, was not mentioned due to the absence of data. However, this aspect deserves to be considered in future clinical practice.

\section{Conclusions}

In conclusion, the present study comprehensively evaluated the clinical values of AFP and DCP for HCC patients from the HBV epidemic area. Positivity of pretreatment AFP and DCP or less than 50\% treatment response rates correlated in higher aggressiveness of $\mathrm{HCC}$, resulting in poor PFS and OS in HCC patients. Therefore, distinguishing different subgroups of patients and selecting the most appropriate therapeutic modality and follow-up intervals for future individualized treatment would be warranted.

\section{Acknowledgments}

All authors have no conflicts of interest to declare. The authors acknowledge and express their deepest gratitude to the participants of this study.

\section{Disclosure}

The authors report no conflicts of interest in this work.

\section{References}

1. Bray F, Ferlay J, Soerjomataram I, et al. Global cancer statistics 2018: GLOBOCAN estimates of incidence and mortality worldwide for 36 cancers in 185 countries. CA Cancer J Clin. 2018;68:394-424. doi: $10.3322 /$ caac. 21492
2. Panel NHC. Hepatobiliary cancers, V.1.2010. Nccn Guidelines. 2019;1:1-142.

3. Omata M, Cheng AL, Kokudo N, et al. Asia-Pacific clinical practice guidelines on the management of hepatocellular carcinoma: a 2017 update. Hepatol Int. 2017;11:317-370.

4. Tateishi R, Yoshida H, Matsuyama Y, et al. Diagnostic accuracy of tumor markers for hepatocellular carcinoma: a systematic review. Hepatol Int. 2008;2:17-30. doi:10.1007/s12072-007-9038-x

6. Imamura H, Matsuyama Y, Miyagawa Y, et al. Prognostic significance of anatomical resection and des- $\gamma$-carboxy prothrombin in patients with hepatocellular carcinoma. $\mathrm{Br} J \mathrm{~J}$ Surg. 1999;86:1032-1038. doi:10.1046/j.1365-2168.1999.01185.x

7. Chon YE, Choi GH, Lee MH, et al. Combined measurement of preoperative $\alpha$-fetoprotein and des- $\gamma$-carboxy prothrombin predicts recurrence after curative resection in patients with hepatitisB-related hepatocellular carcinoma. Int $J$ Cancer. 2012;131:23 32-2341. doi:10.1002/ijc. 27507

8. Kamiyama $\mathrm{T}$, Yokoo $\mathrm{H}$, Kakisaka $\mathrm{T}$, et al. Multiplication of alpha-fetoprotein and protein induced by vitamin $\mathrm{K}$ absence-II is a powerful predictor of prognosis and recurrence in hepatocellular carcinoma patients after a hepatectomy. Hepatol Res. 2015;45:E21E31. doi:10.1111/hepr.12451

9. Li XL, Zhu XD, Cai H, et al. Postoperative $\alpha$-fetoprotein response predicts tumor recurrence and survival after hepatectomy for hepatocellular carcinoma: a propensity score matching analysis. Surgery (United States). 2019;165:1161-1167.

10. Rungsakulkij N, Suragul W, Mingphruedhi S, et al. Prognostic role of alpha-fetoprotein response after hepatocellular carcinoma resection. World J Clin Cases. 2018;6:110-120. doi:10.12998/ wjcc.v6.i6.110

11. Durazo FA, Blatt LM, Corey WG, et al. Des- $\gamma$-carboxyprothrombin, $\alpha$-fetoprotein and AFP-L3 in patients with chronic hepatitis, cirrhosis and hepatocellular carcinoma. $J$ Gastroenterol Hepatol. 2008;23:1541-1548. doi:10.1111/j.1440-1746.2008.05395.x

12. Okuda H, Nakanishi T, Takatsu K, et al. Measurement of serum levels of des-gamma-carboxy prothrombin in patients with hepatocellular carcinoma by a revised enzyme immunoassay kit with increased sensitivity. Cancer. 1999;85:812-818. doi:10.1002/(SICI) 1097-0142(19990215)85:4<812::AID-CNCR8>3.0.CO;2-O

13. Johnson PJ. The role of serum alpha-fetoprotein estimation in the diagnosis and management of hepatocellular carcinoma. Clin Liver Dis. 2001;5:145-159. doi:10.1016/S1089-3261(05)70158-6

14. Liu C, Xiao GQ, Yan LN, et al. Value of $\alpha$-fetoprotein in association with clinicopathological features of hepatocellular carcinoma. World J Gastroenterol. 2013;19:1811-1819. doi:10.3748/wjg.v19.i11.1811

15. Kiriyama S, Uchiyama K, Ueno M, et al. Triple positive tumor markers for hepatocellular carcinoma are useful predictors of poor survival. Ann Surg. 2011;254:984-991. doi:10.1097/SLA.0b013e3182215016

16. Hiraoka A, Michitaka K, Kumada T, et al. Prediction of prognosis of intermediate-stage $\mathrm{HCC}$ patients: validation of the tumor marker score in a Nationwide Database in Japan. Liver Cancer. 2019;8:403-411. doi:10.1159/000495944

17. Nakagawa S, Beppu T, Okabe H, et al. Triple positive tumor markers predict recurrence and survival in early stage hepatocellular carcinoma. Hepatol Res. 2014;44:964-974. doi:10.1111/hepr.12277

18. Yen CW, Kuo YH, Wang JH, et al. Did AFP-L3 save ultrasonography in community screening? Kaohsiung J Med Sci. 2018;34:583-587. doi:10.1016/j.kjms.2018.05.005

19. Yang SL, Liu LP, Yang S, et al. Preoperative serum $\alpha$-fetoprotein and prognosis after hepatectomy for hepatocellular carcinoma. Br J Surg. 2016;103:716-724. doi:10.1002/bjs.10093

20. Hamamura K, Shiina Y, Shiina S, et al. Unique clinical characteristics of patients with hepatocellular carcinoma who present with high plasma des- $\gamma$-carboxy prothrombin and low serum $\alpha$-fetoprotein. Cancer. 2000;88(7):1557-1564. doi:10.1002/(sici)1097-0142(20000 401) $88: 7<1557:$ :AID-CNCR9>3.0.CO;2-G 
21. Gui CH, Baey S, D'cruz RT, et al. Trans-arterial chemoembolization + radiofrequency ablation versus surgical resection in hepatocellular carcinoma - a meta-analysis: TACE+RFA vs Surgery in HCC a Meta-analysis. Eur J Surg Oncol. 2020;46:763-771. doi:10.1016/ j.ejso.2020.01.004

22. Lee YK, Kim SU, Kim DY, et al. Prognostic value of $\alpha$-fetoprotein and des- $\gamma$-carboxy prothrombin responses in patients with hepatocellular carcinoma treated with transarterial chemoembolization. $B M C$ Cancer. 2013;13. doi:10.1186/1471-2407-13-5

23. Liu G, Ouyang Q, Xia F, et al. Alpha-fetoprotein response following transarterial chemoembolization indicates improved survival for intermediate-stage hepatocellular carcinoma. Hpb. 2019;21:10 7-113. doi:10.1016/j.hpb.2018.06.1800

24. Fan J, Wu Z-Q, Zhou J, et al. Hepatocellular carcinoma associated with tumor thrombosis in the portal vein: the effects of different treatments. Hepatobiliary Pancreat Dis Int. 2003;2:513-519.
25. Fan J, Zhou J, Wu ZQ, et al. Efficacy of different treatment strategies for hepatocellular carcinoma with portal vein tumor thrombosis. World J Gastroenterol. 2005;11:1215-1219. doi:10.3748/wjg.v11. i8. 1215

26. Poon RTP, Fan ST, Lo CM, et al. Difference in tumor invasiveness in cirrhotic patients with hepatocellular carcinoma fulfilling the Milan criteria treated by resection and transplantation: impact on long-term survival. Ann Surg. 2007;245:51-58. doi:10.1097/01.sla.0000225255. 01668.65

27. Zhu AX, Kang YK, Yen CJ, et al. Ramucirumab after sorafenib in patients with advanced hepatocellular carcinoma and increased $\alpha$ fetoprotein concentrations (REACH-2): a randomised, double-blind, placebo-controlled, Phase 3 trial. Lancet Oncol. 2019;20:282-296. doi:10.1016/S1470-2045(18)30937-9

\section{Publish your work in this journal}

The Journal of Hepatocellular Carcinoma is an international, peerreviewed, open access journal that offers a platform for the dissemination and study of clinical, translational and basic research findings in this rapidly developing field. Development in areas including, but not limited to, epidemiology, vaccination, hepatitis therapy, pathology and molecular tumor classification and prognostication are all considered for publication. The manuscript management system is completely online and includes a very quick and fair peer-review system, which is all easy to use. Visit http://www.dovepress.com/ testimonials.php to read real quotes from published authors. 